\title{
ENTORNOS DE APRENDIZAJE MÓVILES ADAPTATIVOS Y EVALUACIÓN: COMOLE Y GESES
}

\author{
(ADAPTIVE MOBILE LEARNING ENVIRONMENTS AND EVALUATION: \\ COMOLE AND GESES)
}

\author{
Alvaro Ortigosa \\ Javier Bravo \\ Rosa M. Carro \\ Universidad Autónoma de Madrid (España) \\ Estefanía Martín \\ Universidad Rey Juan Carlos (España)
}

\section{RESUMEN}

En este artículo se presentan los fundamentos y experiencias de uso de dos sistemas que dan soporte a la creación y evaluación, respectivamente, de entornos de aprendizaje móviles adaptativos. En estos entornos, generados dinámicamente por el sistema CoMoLE, se recomiendan las actividades más adecuadas para ser realizadas por cada estudiante en cada momento, facilitándole así el aprovechamiento de su tiempo disponible; también se adapta la interfaz que da soporte a la realización de las actividades, seleccionando los contenidos y herramientas más apropiados en cada caso. Para ello, se consideran las características y necesidades del estudiante, sus acciones previas y el contexto en que se encuentra en ese momento. Sin embargo, es complejo evaluar cuán satisfactoriamente las recomendaciones y adaptaciones atienden las necesidades de cada usuario. Con el objetivo de evaluar entornos de enseñanza adaptativos, se diseñó el método GeSES que, utilizando técnicas de Minería de Datos, extrae, de los logs del sistema adaptativo, información sobre los puntos donde los estudiantes tuvieron mayores dificultades. Este método se ha utilizado para evaluar un entorno generado por CoMoLE. Los resultados obtenidos se presentan también en este artículo.

Palabras clave: evaluación, hipermedia adaptativa, sistemas de recomendación, e-learning. 


\begin{abstract}
In this paper, we present the basis and case of use of two systems that support the creation and evaluation of adaptive mobile learning environments. In this type of environments, dynamically generated by CoMoLE, the most suitable activities to be carried out by each student are recommended to him, so that he can take benefit from his spare time. The interface to support activity accomplishment is adapted by selecting the most suitable contents and tools for that student. With this purpose, student features, needs, previous interactions and context is considered. However, evaluating whether the recommendations and adaptation fits the student's needs is complex. With the purpose of evaluating adaptive learning systems, the method GeSES was designed. GeSES uses Data Mining techniques to extract information about potential problems. It has been used to evaluate one CoMoLE-based learning environment and the results obtained are also presented in this article.
\end{abstract}

Keywords: evaluation, adaptive hypermedia, recommender systems, e-learning.

Desde sus orígenes, Internet se ha utilizado para buscar información especializada con diferentes propósitos (entretenimiento, trabajo, aprendizaje, curiosidad, etc.) y en particular, muchos usuarios utilizan Internet frecuentemente para completar su formación, accediendo a materiales educativos que les permiten aprender a su propio ritmo. Más aún, es cada vez más frecuente que las propias instituciones educativas, especialmente las universidades, basen parte de sus actividades en recursos educativos accesibles a través de Internet.

Sin embargo, la heterogeneidad de la información disponible a través de Internet provoca que no todos los recursos sean adecuados para todos los usuarios, ya que cada persona puede tener unas necesidades, preferencias y objetivos diferentes a las del resto. Así, cada vez son más los sitios Web que incluyen funcionalidad para poder adaptar sus contenidos a distintos tipos de usuarios según sus características. En particular, los Sistemas Hipermedia Adaptativos (SHA) (Brusilovsky et al., 1998) son sistemas que, basándose en un modelo del usuario, personalizan la información que presentan considerando la información contenida en ese modelo.

Por otra parte, gracias a la disponibilidad hoy en día de tecnologías inalámbricas y a la rápida evolución de los dispositivos móviles, es posible conectarse a Internet desde distintos lugares y en diferentes momentos. Los dispositivos móviles son pequeños, compactos y portátiles. Cada vez es mayor la cantidad de personas que llevan consigo asiduamente uno o más dispositivos electrónicos, incluyendo teléfonos, asistentes personales digitales (PDAs) y ordenadores portátiles. Estos 
dispositivos se suelen utilizar con fines de entretenimiento, para trabajar o para aprender. Suelen tener diversas prestaciones como cámara y reproductor de vídeo, sistema de localización física como GPS, capacidad para capturar datos, etc. Además, en la sociedad actual, el tiempo se ha convertido en un valor muy preciado, y en muchos casos organizar el tiempo disponible de una forma óptima es complicado, aunque necesario.

En este contexto, las tecnologías inalámbricas y los dispositivos móviles ofrecen una gran oportunidad a quienes desean aprovechar espacios de tiempo libre para realizar tareas pendientes cuando no tienen acceso a su ordenador personal. Conviene considerar que las actividades a realizar en distintas situaciones pueden depender de las características de los dispositivos móviles que se tienen al alcance en ese momento, así como del lugar en que se encuentre el usuario y, icómo no! del tiempo que tiene disponible. Por tanto, sería útil disponer de entornos de recomendación de actividades a los que los usuarios se puedan conectar para pedir consejo sobre las actividades a realizar en distintos contextos utilizando distintos dispositivos.

Desde el punto de vista de diseño, la creación y configuración de entornos de enseñanza y aprendizaje adaptativos móviles que puedan dar soporte a la recomendación y realización de actividades por parte de cada usuario en función de sus características personales, necesidades, acciones previas y contexto, es una labor complicada. Esto implica la necesidad de: especificar y dar soporte a la realización de distintos tipos de actividades; crear y ofrecer distintas versiones de contenidos para seleccionar los más apropiados para usuarios con distintas necesidades y en distintos contextos; y definir criterios de recomendación de actividades y de adaptación de contenidos para la generación de espacios de trabajo adaptados a las necesidades de los usuarios. Todas estas tareas requieren un tiempo y esfuerzo notables por parte del diseñador o creador de este tipo de entornos. Actualmente, los sistemas hipermedia adaptativos no suelen incluir ayudas para definir y combinar distintos atributos y criterios de adaptación sobre usuarios, grupos de trabajo, actividades y contextos, a ser considerados para realizar recomendaciones y generar espacios de trabajo dinámicamente. Son pocos los sistemas de recomendación que proveen a los diseñadores herramientas y recursos que puedan utilizarse de forma sencilla para reducir el tiempo empleado en la creación y configuración de SHA.

Es en este contexto donde se sitúa el sistema CoMoLE. ("Context-based adaptive Mobile Learning Environments"). CoMoLE da soporte a la recomendación y realización de actividades desde distintos dispositivos a través de navegadores Web, considerando las necesidades de cada usuario y el contexto en el que se encuentra en cada momento. 
Aunque los entornos de e-learning ofrecen mayor interactividad y mayor flexibilidad, su mantenimiento representa un importante obstáculo para su utilización por parte de los profesores. Además de diseñar e implementar las actividades educativas, evaluar a los estudiantes y ofrecer apoyo normalmente en forma de tutorías, estos profesores deben evaluar el efecto de las distintas actividades sobre el aprendizaje de los estudiantes, es decir, intentar conocer si éstas fueron útiles para los estudiantes, y de qué manera se podrían mejorar. Esta evaluación es especialmente importante cuando no todos los estudiantes reciben los mismos recursos de la misma forma, sino que éstos son adaptados a sus características y contextos, como es el caso de los SHA.

En este sentido, el principal obstáculo al que se enfrentan los profesores para llevar a cabo la evaluación es que, mientras en la enseñanza tradicional el profesor percibe mediante observación directa las reacciones y comportamientos de los estudiantes, pudiendo adaptar la forma en la que transmite los conocimientos en función de lo observado, en el caso de los entornos de e-learning el profesor no puede obtener este tipo información de la misma forma, ya que generalmente no está físicamente presente en el momento en el que los estudiantes realizan estas actividades ni puede observar remotamente a cada estudiante en cada momento. Esto hace aún más compleja la tarea de evaluación de las actividades propuestas y de las decisiones de adaptación adoptadas. Una posibilidad para facilitar la evaluación consiste en incorporar cuestionarios para que los estudiantes puedan opinar explícitamente sobre los recursos a los que han accedido. Sin embargo, esta solución presenta varias dificultades: muchas veces los estudiantes no disponen del tiempo o la voluntad para rellenar encuestas; es posible que los estudiantes no quieran expresar opiniones negativas sobre los recursos; e incluso, aunque lo hagan, sus percepciones pueden no ser correctas. Por estos motivos; sería mejor poder disponer de información más objetiva sobre lo adecuadas que resultaron las recomendaciones, de las diversas actividades y los recursos presentados en cada momento.

Es práctica común que estos entornos almacenen las interacciones de los estudiantes en bases de datos relacionales o ficheros (logs). Estas interacciones reflejan lo que hicieron los estudiantes, ya que contienen información sobre sus acciones, progresos y resultados obtenidos en cada una de las actividades realizadas. Por tanto, el profesor podría utilizar los datos contenidos en los logs como fuente de información sobre la experiencia de sus estudiantes con las actividades educativas. Esta información no es evidente, pero existe la posibilidad de obtenerla mediante un análisis de estos logs. No obstante, el tamaño de los logs, normalmente es excesivo y su análisis no es posible sin la ayuda de métodos y herramientas adecuadas para esta tarea. 
La minería de datos es la disciplina capaz de extraer información implícita contenida en los datos, que previamente es desconocida, y además es potencialmente útil (Witten y Frank, 2000). Esta disciplina proporciona, un conjunto de métodos y técnicas que han demostrado su capacidad para analizar grandes cantidades de datos con éxito en ámbitos como el comercio electrónico. Una posibilidad para conseguir analizar los logs en entornos adaptativos, consiste en que el profesor utilice técnicas de minería de datos. Sin embargo, si la aplicación de estas técnicas y la posterior interpretación de los resultados obtenidos no son tareas sencillas incluso para expertos en esta disciplina, lo son mucho menos para profesores que a veces sólo poseen conocimientos básicos de informática.

Por este motivo, se propone un método de evaluación para este tipo de entornos de recomendación móviles que ayuda a realizar el análisis de logs y ofrece los resultados de este análisis de forma entendible. Este método fue aplicado a los logs del sistema CoMoLE con el objetivo de detectar situaciones problemáticas que pueden indicar una baja eficacia en las recomendaciones de actividades realizadas por este sistema.

Además, en este trabajo se presentan las bases del sistema CoMoLE, junto con un ejemplo de funcionamiento y los resultados de experiencias realizadas con dos entornos de aprendizaje móvil para el apoyo al estudio de sendas asignaturas de primer y segundo curso de Ingeniería Informática de la Universidad Autónoma de Madrid. Igualmente, se presenta el método GeSES para la evaluación de cursos adaptativos, mientras que se muestra un ejemplo de su aplicación al análisis de logs del sistema CoMoLE. Se describen algunos trabajos relacionados con las áreas de las propuestas, y finalmente se presentan las conclusiones.

\section{EL SISTEMA CoMoLE}

Con el objetivo de ofrecer a los estudiantes la posibilidad acceder a actividades educativas desde distintos dispositivos, y que estas actividades se adapten tanto a su perfil de usuario como al contexto en que se encuentran, se desarrolló el sistema CoMoLE. (Context-based adaptive Mobile Learning Environments). Este sistema recomienda las actividades más apropiadas a cada usuario en cada momento, dependiendo de sus características personales, de las acciones mientras interactúa con la plataforma y del contexto en el que se encuentra en ese momento. Estas actividades serán realizadas a través de un navegador Web utilizando distinto tipo de dispositivos. A continuación se presentan las bases de este sistema, seguidas de un ejemplo de funcionamiento y, por último, algunos resultados de experiencias de uso realizadas con este sistema. 
A. Ortigosa; J. Bravo; R. Carro; E. Martín

Entornos de Aprendizaje Móviles Adaptativos y Evaluación: Comole y Geses

\section{Bases de CoMoLE}

Un requisito fundamental para poder recomendar actividades a cada usuario y generar dinámicamente las interfaces que den soporte a su realización en cada contexto, es almacenar información sobre los usuarios que las llevarán a cabo. Estos datos constituirán el modelo de usuario de CoMoLE. En este modelo de usuario es posible definir cualquier rasgo del usuario cuyos posibles valores puedan representarse a través de un conjunto de valores discretos (numéricos o estereotipos) o mediante intervalos de valores en el caso de datos numéricos. Algunos ejemplos de rasgos de adaptación utilizados en el área de la enseñanza son si el estudiante cursa por primera vez una materia o no, su nivel de conocimiento previo, las distintas dimensiones del estilo de aprendizaje del usuario, el porcentaje de aciertos en los ejercicios realizados hasta el momento, el lugar donde se encuentra, el dispositivo utilizado, o el tiempo que tiene disponible para realizar las actividades, por ejemplo.

Una vez que se han definido las características de los usuarios que se van a tener en cuenta a la hora de realizar la adaptación, es necesario describir los entornos de aprendizaje móviles, especificando sus componentes. Un entorno de este tipo consiste en: un conjunto de actividades, relacionadas con un tema, que se recomendarán a usuarios en distintas situaciones; un conjunto de contenidos multimedia y herramientas que se combinarán para dar lugar a espacios de trabajo en los que los usuarios podrán realizar dichas actividades; y un conjunto de parámetros y criterios de recomendación de actividades y adaptación de espacios de trabajo. Para cada entorno se definen una serie de características generales como: I) idiomas a los que se dará soporte en el entorno (contenidos, interfaz),II) descripciones del propio entorno en los distintos idiomas que se hayan especificado, III) atributos de los usuarios que se van a tener en cuenta para realizar la recomendación de actividades y adaptación de espacios de trabajo, IV) actividades a realizar, V) roles que los estudiantes pueden tener en las actividades colaborativas, y VI) características generales de los grupos de trabajo, como por ejemplo si los estudiantes serán agrupados de forma automática, el tamaño de los grupos de trabajo o el conjunto de herramientas que se facilitarán a los estudiantes para poder interactuar entre sí y realizar las actividades colaborativas.

También se almacena información sobre cada una de las actividades que forman parte de un entorno, como el tipo de actividad (teoría, ejemplo, simulación, ejercicio tipo test, ejercicio de respuesta libre, repaso, envío/recepción de mensajes, etc); una descripción de la actividad por cada uno de los idiomas especificados en las características generales del entorno a la que pertenece; fecha de inicio/finalización de la actividad ó plazo límite (si procede); tiempo mínimo estimado de realización de la actividad (si existe) y tiempo máximo que el estudiante puede tardar en realizar 
la actividad desde su inicio (si procede). Esta información se almacena en el caso de actividades atómicas, es decir, aquellas que no se descomponen en actividades más simples. Dentro de estos entornos, también hay actividades compuestas cuyo principal objetivo es agrupar conjuntos de actividades, por ejemplo, aquellas que se relacionen con un mismo concepto.

Además, las actividades atómicas pueden tener asociados contenidos multimedia. Los contenidos asociados a cada actividad pueden variar dependiendo del tipo de la misma, del tipo de dispositivo para el cual están diseñados, del estilo de aprendizaje del estudiante y/o de otras características personales; de este modo, se podrán seleccionar los más adecuados para cada usuario en cada situación particular. En el caso de actividades teóricas o de ejemplos, los contenidos podrán ser fragmentos HTML, XML o ficheros PDF con explicaciones sobre un concepto o casos de ejemplos a observar. En los ejercicios, los contenidos serán el enunciado del problema a solucionar. En las simulaciones, se pueden incluir applets con los que los usuarios podrán interactuar para observar distintos comportamientos del problema. En los mensajes enviados entre compañeros del mismo grupo de trabajo, los contenidos serán el cuerpo del mensaje a enviar. Y por último; en los envíos o descargas de ficheros de material, los contenidos serán el propio fichero. Independientemente del tipo de actividad, cada uno de los contenidos podrá disfrutar de distinto tipo de material, como imágenes, texto, vídeos o sonidos, entre otros. Un mismo contenido puede estar asociado a distintas actividades. Una misma actividad puede tener asociadas distintas versiones de contenidos orientadas a usuarios con características o necesidades diferentes (por ejemplo, versiones con más esquemas y menos textos para estudiantes con estilo de aprendizaje visual, versiones con vocabulario más sencillo para estudiantes noveles, etc.). El diseñador o creador de contenidos puede crear o suministrar diferentes contenidos dependiendo del tipo de dispositivo utilizado por el usuario. Por ejemplo, en una determinada actividad, se pueden definir distintos contenidos, unos para el caso en el que el usuario utilice un ordenador personal o portátil y otros para cuando se conecte con una PDA. En este caso, lo más usual es que los contenidos mostrados a los usuarios si utilizan una PDA se encuentren más sintetizados o se muestre la misma información que cuando se utiliza un ordenador personal, pero dividiéndola en varias páginas, para que el usuario pueda visualizar dichos contenidos de una forma más cómoda. También se pueden definir distintas versiones de contenidos dependiendo de atributos de los usuarios como, por ejemplo, la dimensión visual-verbal de los estilos de aprendizaje de los alumnos (Felder y Silverman, 1988): en determinadas actividades, puede ser útil disponer de dos versiones distintas, una para estudiantes con estilo de aprendizaje visual, donde las explicaciones serán más esquemáticas y contendrán más dibujos y gráficos, y otra con explicaciones textuales más extensas para los 
estudiantes con estilo de aprendizaje verbal. Además, se pueden ofrecer distintas explicaciones dependiendo de la experiencia del usuario adaptando el vocabulario o el tipo de expresiones de las distintas versiones de contenidos, por ejemplo.

Una vez que se ha establecido el modelo de usuario y las características del entorno de trabajo y de sus actividades; el siguiente paso es, definir los criterios para recomendar las distintas actividades a los distintos usuarios y grupos teniendo en cuenta los rasgos definidos en el modelo de usuario, así como las características de las actividades. Estos criterios se establecerán por medio de reglas de adaptación. Las reglas de adaptación pueden tener asociadas condiciones de activación, relacionadas con información sobre los usuarios. Si una regla no tiene condición de activación se aplicará automáticamente para todos los usuarios. En caso contrario, es necesario que la condición se satisfaga para que la regla se active. Para todos los tipos de reglas de recomendación, las condiciones de activación pueden ser simples o compuestas. Una condición está definida por un nombre de atributo y un valor constante con el que se comparará, a través de un operador de relación, el valor de dicho atributo en el modelo de usuario del estudiante. En el caso de atributos numéricos, el operador de relación podrá ser el de igualdad, mayor, mayor e igual, menor o menor igual. Sin embargo en el caso de atributos cuyo valor sea de tipo discreto (ya sea numérico o estereotipo) el operador de relación a utilizar será siempre el de igualdad. Las condiciones compuestas tienen condiciones anidadas, que a su vez pueden ser simples o compuestas. Para definir relaciones entre las condiciones anidadas se pueden utilizar dos clases de operadores: "y" u "o". Además, pueden especificarse condiciones negativas, en las que una regla se activa cuando no se cumple una determinada condición, ya sea simple o compuesta. Las reglas de adaptación pueden ser de tres tipos: reglas estructurales, filtros generales de contexto y, reglas de requisitos individuales.

Las reglas estructurales, indican la manera en que se descomponen las actividades compuestas en otras actividades a las que llamamos subactividades, así como la flexibilidad de la guía de navegación ofrecida a los distintos usuarios durante la realización de dichas subactividades (recorrido secuencial/libre), incluyendo el orden en el que los usuarios tendrán que realizarlas, en el caso que la guía sea directa (recorrido secuencial). Una actividad puede descomponerse de distintas formas, e incluso la guía de navegación ofrecida entre las subactividades puede ser distinta dependiendo del tipo de usuario a quien esté dirigida. De este modo, es posible guiar de forma distinta a usuarios diferentes mientras realizan las subactividades de una misma actividad. Una versión más simple de este tipo de reglas se ha utilizado previamente para describir cursos hipermedia adaptativos (Carro, 2001). 
Los filtros generales de contexto, sirven para separar, del conjunto de todas las actividades, aquellas que se considera apropiado recomendar al usuario debido al contexto en el que se encuentra en el momento de ofrecer la recomendación, así como aquellas que se consideran inapropiadas por el mismo motivo. En estos filtros también es posible considerar características personales de los usuarios para ofrecer las recomendaciones, de modo que es posible ofrecer distintas recomendaciones a usuarios distintos, incluso cuando se encuentran en el mismo contexto. Por ejemplo, se podría definir un filtro general de contexto con el siguiente criterio: "Las actividades teóricas y los ejemplos no serán recomendadas a los estudiantes que tengan menos de treinta minutos disponibles". Además, se podría especificar otro filtro general que indicara que, si se tienen 10 minutos libres, las actividades de repaso y los ejercicios tipo test sí se recomendarán a los usuarios de estilo de aprendizaje activo.

Por último, las reglas de requisitos individuales, especifican restricciones de realización para una determinada actividad. Este tipo de reglas se pueden utilizar para especificar, por ejemplo: el número mínimo de usuarios necesarios para poder realizar la actividad; si es necesario la realización de una o varias actividades relacionadas previamente; la fecha de activación de la actividad; o la obligatoriedad de que se cumplan una serie de condiciones del contexto de los usuarios, entre otros.

A veces, la especificación de todo este tipo de criterios de adaptación es un proceso complicado para el responsable del entorno. Además, en determinadas ocasiones, puede ocurrir que no exista información suficiente sobre la adecuación de una determinada actividad para un usuario concreto que se encuentra en un contexto específico; es decir, no existen reglas de recomendación que indiquen si recomendar o no esa actividad. En este caso, se pueden realizar recomendaciones basadas en la información sobre las acciones que otros usuarios con características similares realizaron previamente. Evidentemente, para poder ofrecer este tipo de recomendaciones basadas en las acciones de otros usuarios con características y contextos similares, es necesario almacenar y procesar información sobre usuarios que interactuaron con el entorno previamente. En caso de no existir información disponible para una actividad determinada, CoMoLE. Anota dicha actividad como disponible y se deja en manos del usuario realizarla o no.

Para la realización de las recomendaciones basadas en información de otros usuarios, CoMoLE. Utiliza modelos de Markov. El primer paso consiste en realizar una clasificación de usuarios en función de los rasgos de adaptación que se tienen en cuenta en todo el entorno, para ofrecer las recomendaciones. Cada usuario está definido por un vector de atributos, que pueden estar relacionados tanto con sus rasgos personales como con características de su contexto. Cada elemento del vector 
tendrá asignado el valor del atributo correspondiente para ese usuario. En función de los posibles valores para cada atributo del vector, realizando todas las combinaciones de valores para cada atributo, se obtiene un conjunto de vectores, cada uno de los cuales representa una clase diferente de usuarios.

Consideremos un entorno en el que se tienen en cuenta la dimensión secuencialglobal de los estilos de aprendizaje de los alumnos y el dispositivo que utilizan para realizar actividades de ese entorno; distinguiendo entre ordenador personal/ portátil y, dispositivo móvil (PDA/teléfono móvil). En este caso concreto, hay cuatro posibles clases de usuario: secuencial utilizando un ordenador personal o portátil (tipo 1), secuencial utilizando PDA o teléfono móvil (tipo 2), global utilizando un ordenador (tipo 3) y global utilizando una PDA o teléfono (tipo 4). En el caso de atributos numéricos (como, por ejemplo, el tiempo disponible), los valores posibles son demasiados como para poder obtener suficientes usuarios de cada una de las categorías en función de los minutos que tienen disponibles. Por este motivo, se ha decidido agrupar por rangos los posibles valores en este tipo de atributos.

Para cada una de estas clases de usuarios, se puede obtener un recorrido de actividades con información sobre la probabilidad de realización de una determinada actividad después de haber realizado otra por parte de usuarios con las características asociadas a la clase. El recorrido de actividades para cada clase de usuarios se obtiene analizando el orden en el que los usuarios de esta clase realizaron las actividades del entorno. Cada recorrido contiene información sobre todas las actividades que forman parte del entorno y cuántos usuarios de esa clase realizaron una determinada actividad después de otra. Estos recorridos de actividades se han representado utilizando un modelo de Markov donde los estados representan las actividades del entorno y los enlaces entre las mismas indican la probabilidad de que un usuario realice la actividad en un momento determinado.

Una vez obtenidos los recorridos realizados entre las actividades, para cada una de las clases de usuario, se puede decidir si recomendar o no una determinada actividad teniendo en cuenta el tipo de usuario involucrado, la última actividad realizada (que será considerada como la actividad origen), la actividad sobre la que se desea obtener información (actividad destino), los recorridos entre actividades realizados por usuarios similares (pertenecientes a la misma clase), y la relación que existe entre las probabilidades de transición desde la actividad origen a las posibles actividades destino.

La recomendación sobre una determinada actividad, se realiza si se puede sugerir con suficiente confianza. Se considera que existe suficiente confianza 
sobre la probabilidad de una transición cuando la probabilidad de transición de la actividad origen a la actividad destino con respecto a cada una de las demás posibles actividades destino, es superior a un 30\%. En ese caso, la actividad destino inicial se anota como recomendada para ese usuario, ya que es mucho más probable que le interese esta frente a las otras.

Por ejemplo, observando la figura 1, supóngase que se trata de un usuario con una serie de características que acaba de realizar la actividad BA_Gates. El responsable del entorno no especificó ninguna regla de adaptación relacionada con las actividades Set_Tests y Set_Exers y, por tanto, no se tiene información sobre la adecuación de dichas actividades. Si se suponen las probabilidades de transición presentadas en el gráfico de la figura 1, se tiene que se recomendará al usuario la actividad Set_Tests, ya que un $75 \%$ de los usuarios con características similares en contextos análogos realizaron esta actividad a continuación de la actividad BA_Gates. Sin embargo, no existe información sobre la adecuación de la actividad Set_Exers para este tipo de usuarios en estos contextos. Por tanto, esta última actividad no será especialmente recomendada, dejando su realización en manos.

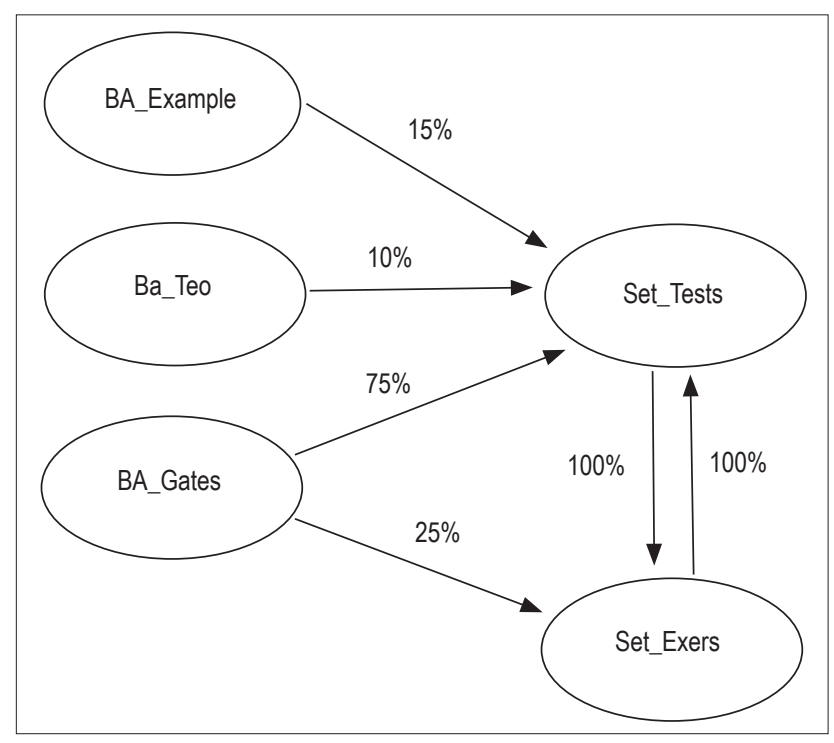

Figura 1. Ejemplo de un modelo de Markov con las probabilidades de transición entre distintas actividades de un entorno 
Tanto el mecanismo de recomendación basado en reglas de adaptación como el basado en información sobre otros usuarios se encuentran implementados en dos módulos independientes del sistema CoMoLE. Este hecho permite que puedan utilizarse de forma independiente.

Cuando un entorno de aprendizaje móvil, se pone a disposición de los usuarios, éstos tienen que acceder utilizando un navegador Web al sistema. El primer paso consiste en averiguar cuáles son las actividades relacionadas con el entorno seleccionado por el usuario. El gestor de actividades es el responsable de obtener un listado inicial de las actividades de un entorno determinado. Para generarlo, el gestor consulta tanto la información sobre la totalidad de los elementos del entorno como la información sobre las actividades que ya ha realizado el usuario. A partir de esto, obtiene el listado inicial de actividades, anota las realizadas previamente por el usuario, y proporciona un listado inicial que envía al módulo de recomendación basado en reglas.

A continuación, el listado inicial pasa por los tres filtros descritos en el capítulo anterior: filtro estructural, filtro general de contexto y, filtro individual. Cada módulo da soporte al procesamiento del correspondiente tipo de reglas: reglas estructurales, filtros generales de contexto y restricciones individuales de realización. Esta separación de filtros en módulos facilita que se pueda utilizar cada uno de ellos por separado, de tal manera que, en función de las decisiones tomadas por el responsable del entorno en la fase de diseño, se pongan en marcha los correspondientes módulos de recomendación para la fase de recomendación.

La entrada de cada módulo de recomendación es la salida del módulo anterior, a excepción del primero a utilizar, que recibe el listado inicial proporcionado por el gestor de actividades. La salida de cada módulo es un listado con las actividades anotadas junto con su correspondiente grado de recomendación (recomendada en este momento, disponible pero no recomendada en este momento, disponible y no disponible). Este listado es la entrada al módulo de recomendación basado en información sobre otros usuarios, en caso de utilizarse.

En caso de haber seleccionado el uso de recomendaciones basadas en acciones previas de otros usuarios, entra en juego el módulo denominado "buscador de recomendaciones". Su objetivo principal es realizar recomendaciones sobre las actividades que se encuentren sin anotar o bien en estado disponible (ni recomendadas ni no recomendadas) basándose en las actividades realizadas por usuarios con características similares cuando se encontraron en la misma situación. 
Para ello, primeramente, se clasifica al usuario en uno de los tipos o clases para los que se encuentran definidos los grafos de actividades. A continuación, para cada una de las actividades a considerar, el buscador de recomendaciones analiza la probabilidad de transición desde actividades que el usuario ya ha realizado a aquellas actividades a considerar. Si existe suficiente nivel de confianza sobre la probabilidad de realizar una determinada actividad a continuación de una de las actividades realizadas ya por el usuario, la actividad destino es anotada como recomendada.

Una vez que el buscador de recomendaciones ha terminado de actualizar el estado de las actividades, envía la lista de actividades completa anotada al generador de espacios de trabajo, encargado de componer estos espacios seleccionando los contenidos y herramientas más adecuados para mostrar al usuario. Realiza esta selección teniendo en cuenta las características del dispositivo y los rasgos personales de los usuarios, e incluye una tabla de actividades anotada con información sobre el grado de recomendación de cada actividad. Una vez que se ha generado la página, es enviada al navegador Web del usuario.

En la figura 2 se presenta un ejemplo de una página Web generada para un usuario que está interactuando con un entorno de recomendación de actividades relacionado con la materia "Estructura de Datos y de la Información I (EDI1)". Como se puede observar, la parte superior de esta página muestra el área de recomendación donde, en la parte izquierda, se pueden ver las actividades recomendadas (If.Test) y, en la parte derecha, la actividad actual (Switch.Teoría). En la parte central se encuentran dos botones. El superior permite salir del entorno finalizando la sesión. El inferior permite ocultar/mostrar la tabla anotada de actividades (parte izquierda de la página) para ampliar/reducir el tamaño del área de contenidos (parte central y principal de la página). A la izquierda de la página se muestra una tabla de actividades anotadas en distintos colores. Esta tabla se genera dinámicamente a partir del listado de actividades anotadas de los módulos de recomendación.

Como se puede observar, cada actividad está coloreada de acuerdo a la metáfora del semáforo (Weber y Specht, 1997), donde cada uno de los colores indica un grado de recomendación. Los colores utilizados son:

- Verde para indicar que la actividad se encuentra disponible para el usuario y que su realización está recomendada por el entorno en ese momento.

- Amarillo cuando la actividad está disponible pero no se encuentra recomendada en ese momento, ya que alguna condición relacionada con el contexto del usuario no se satisface. 
- Rojo si la actividad no se encuentra disponible y, por tanto, el usuario no puede realizarla (por ejemplo, si es necesario que realice otra actividad previamente).

- Negro para las actividades ya realizadas por el usuario.

En el ejemplo de la figura 2, la tabla de actividades anotada tiene una actividad recomendada que es la actividad If-Test que también se encuentra en la parte superior de la página, en la barra de recomendaciones. La actividad Switch-Ejercicio1 se encuentra anotada como no recomendada en color amarillo ya que alguna de las condiciones del contexto del usuario no son apropiadas para la realización de la misma. La actividad Switch-Ejercicio2 se encuentra anotada como no disponible.

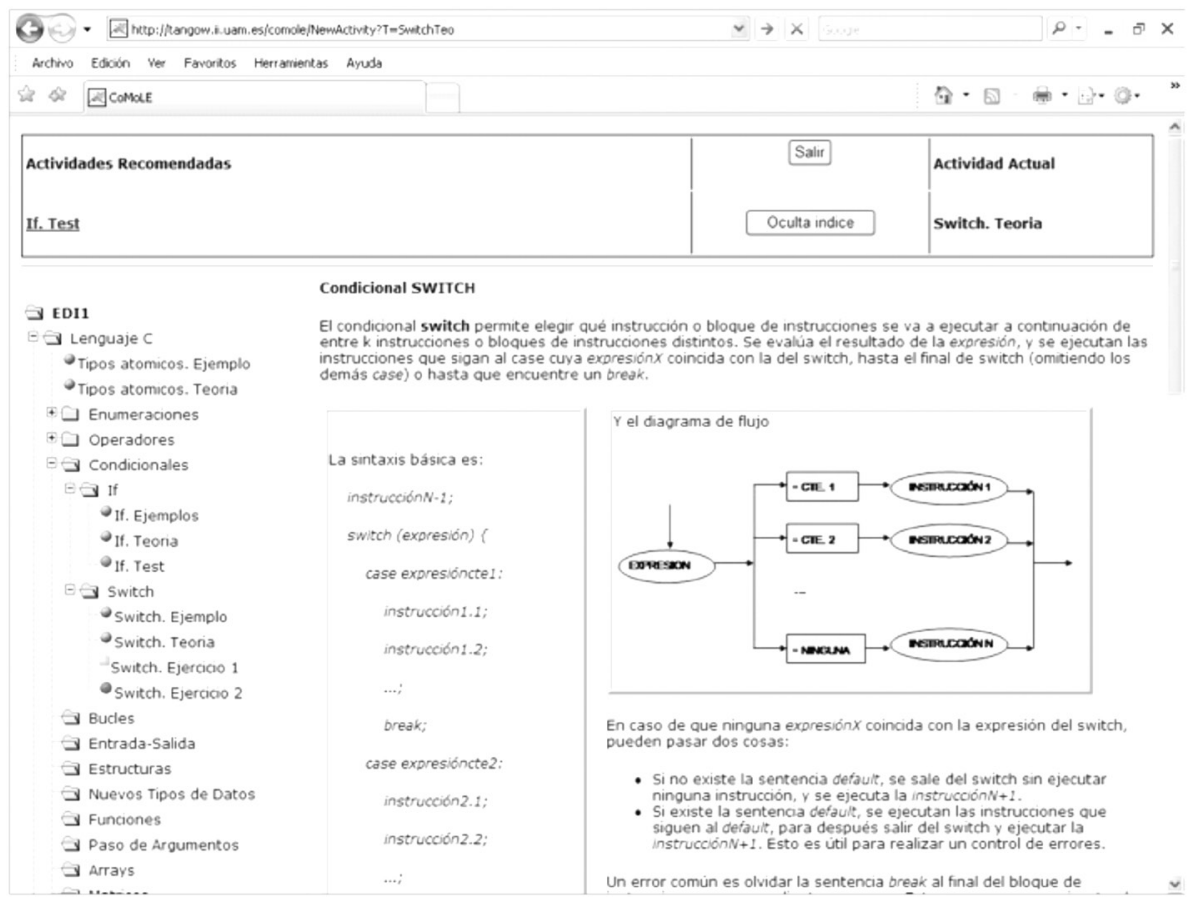

Figura 2. Captura de ejemplo de página Web generada por CoMoLE

Por último, en la parte inferior derecha de la pantalla, se muestra el área con los contenidos y herramientas más adecuadas para realizar la actividad actual (Switch-Teoría en este caso). Los contenidos son seleccionados por el generador de espacios de trabajo teniendo en cuenta tanto las distintas versiones de los contenidos almacenadas en el entorno como los atributos personales del usuario, sus acciones y 
el contexto en que se encuentra (por ejemplo, se seleccionan contenidos textuales/ gráficos según la dimensión visual/verbal de su estilo de aprendizaje o contenidos para PC/PDA dependiendo del dispositivo que esté utilizando el usuario).

\section{Experiencias de uso}

Con el objetivo de comprobar el impacto y la utilidad de la propuesta en entornos adaptativos móviles reales, se llevaron a cabo dos casos de estudio con usuarios de Ingeniería Informática de la Universidad Autónoma de Madrid. Se crearon dos entornos de aprendizaje móvil, uno para el apoyo al estudio de la asignatura de "Estructuras de Datos de la Información I" (en adelante, EDI1) y otro para el apoyo al estudio de "Sistemas Operativos I" (en adelante, SO1), correspondientes al primer y segundo curso respectivamente. Ambas asignaturas se imparten durante el segundo cuatrimestre del curso. Los alumnos utilizaron estos entornos como recursos educativos adicionales a las clases de teoría y prácticas que se impartían presencialmente. Para cada uno de los entornos se definieron distintas actividades de aprendizaje de acuerdo al programa de cada asignatura. El sistema CoMoLE. Dio soporte a la recomendación y realización de actividades en ambos entornos.

Los estudiantes de ambas asignaturas dieron su opinión sobre el sistema y facilitaron comentarios sobre distintos aspectos de estos entornos de recomendación. La información fue recopilada a través de formularios disponibles mientras se encontraban interactuando con el entorno de aprendizaje móvil, y también mediante una encuesta final detallada para recoger más datos sobre su experiencia con el sistema.

El número de estudiantes matriculados en las asignaturas EDI1 y SO1 durante el curso 2007/08 fue 285 y 230 estudiantes respectivamente. Estos entornos fueron utilizados por 135 estudiantes en EDI1 y por 160 alumnos en SO1 (47\% y 69\% de participación con respecto a los estudiantes matriculados, respectivamente). Con el objetivo de motivar a los estudiantes a que se conectaran y de permitirles que se beneficiaran del proceso de recomendación de actividades basado en su contexto, se prestaron 20 PDAs a los estudiantes que no tenían una propia.

El entorno creado para "Estructura de Datos y de la Información I" se centra en actividades de aprendizaje relacionadas con estructuras de datos y programación en lenguaje C. Los profesores habían explicado previamente los temas en clase. Los parámetros de adaptación definidos para este curso son el estilo de aprendizaje (dimensiones visual-verbal, activo-reflexivo y sensorial-intuitivo) y su contexto 
(dispositivo utilizado, tiempo disponible y localización física). El temario de la asignatura "Sistemas Operativos I" consta de nueve temas. El entorno ofrecido para dicha asignatura incluye actividades correspondientes a uno de los temas ("Gestión de Memoria"). Los atributos a tener en cuenta para realizar la recomendación de actividades y adaptación de contenidos en este caso fueron los mismos que en EDI1.

En ambos entornos se incluyen actividades atómicas y actividades compuestas. Estas últimas son utilizadas solamente con fines estructurales, para poder organizar las actividades atómicas, por lo que no tienen ningún contenido asociado. El entorno de EDI1 consta de 133 actividades diferentes, de las cuales 95 son atómicas. En el caso de SO1, hay 79 actividades atómicas distintas de las 91 que componen el conjunto total para este entorno. Además, se definieron varias reglas estructurales para especificar el modo en que se organizarían las actividades para cada tipo de estudiante según la dimensión sensorial-intuitivo de su estilo de aprendizaje.

Las actividades y los contenidos son recomendados a los estudiantes según los rasgos de adaptación considerados en cada asignatura, teniendo en cuenta también información dinámica sobre las acciones que realizaron previamente, como actividades realizadas o resultados obtenidos en los ejercicios.

Cuando se llevaron a cabo estos dos casos de estudio, el módulo de recomendación basada en las acciones de otros usuarios (utilizando modelos de Markov) todavía no estaba implementado por completo. Por tanto, en ambos casos las recomendaciones se basaron en reglas.

Estos dos casos de estudio arrojaron información sobre: la utilidad de los entornos de recomendación basados en las características personales y en el contexto de los usuarios; la calidad de las recomendaciones ofrecidas; la preferencia de sistemas de recomendación frente a sistemas sin ningún tipo de adaptación; el seguimiento de las recomendaciones sugeridas por el sistema; la adecuación de la adaptación de las versiones de contenidos a los perfiles de los estudiantes y al dispositivo que estaban utilizando; la facilidad de uso del sistema; la influencia del sistema en la organización del tiempo de estudio; la intención de los alumnos de volver a utilizar el sistema; cómo se sintieron trabajando con estos nuevos entornos de recomendación; sus opiniones sobre la experiencia y posibles mejoras a realizar sobre el sistema.

En general, los alumnos prefieren entornos de recomendación que tengan en cuenta sus características personales, sus acciones y su contexto, frente a sistemas no adaptativos. Este hecho viene marcado por las características de este tipo de 
entornos, ya que ayudan a los usuarios a escoger las actividades más adecuadas a realizar en cada momento, a conocer con qué conceptos tienen dificultades de aprendizaje, a gestionar su tiempo libre, etc.

Según las opiniones vertidas por los usuarios, las recomendaciones que el sistema realizó fueron adecuadas en la mayoría de los casos, y la gran parte de los estudiantes siguieron las recomendaciones que se les ofrecían en cada paso. El proceso de recomendación que lleva a cabo el sistema CoMoLE. Ayuda a los estudiantes en la selección de actividades a realizar en cada contexto.

Los estudiantes consideraron útiles las recomendaciones basadas en sus características personales, acciones previas y contexto. La adaptación basada en sus características personales (incluyendo estilos de aprendizaje) y nivel de conocimiento fue considerada más importante que la adaptación de contenidos al dispositivo. Dentro de la información de contexto, el factor mejor valorado por la mayoría de los usuarios para ser considerado con propósitos adaptativos es el tiempo. CoMoLE. Les ayuda a gestionar el tiempo disponible para realizar actividades y se convierte en un factor de especial importancia cuando disponen sólo de unos pocos minutos.

En los casos de estudio llevados a cabo, el proceso de recomendación se basó principalmente en la adaptación al contexto del usuario, a su estilo de aprendizaje (dimensiones sensorial-intuitivo y visual-verbal) y a los resultados obtenidos en actividades previas. Sin embargo, aparte de estos rasgos, los comentarios ofrecidos por los estudiantes indican que sería conveniente tener en cuenta también la dimensión global-secuencial de los estilos de aprendizaje junto con el nivel de conocimientos. De esta manera, los alumnos con estilo de aprendizaje global podrían tener más libertad a la hora de navegar entre actividades y los alumnos con conocimientos más avanzados podrían saltar las actividades más básicas.

Sin embargo, al hablar de la adaptación de contenidos, esta situación cambia bastante. Los estudiantes consideran menos importante la adaptación de los espacios de trabajo, ya que para ellos lo realmente importante son los contenidos. Habrían preferido que las actividades cubriesen más parte o la totalidad del temario antes que el hecho de que los contenidos fueran adaptados a la dimensión visual-verbal de sus estilos de aprendizaje o al dispositivo que estaban utilizando.

El poder realizar actividades sin necesidad de estar frente a su ordenador personal fue una posibilidad atractiva pero no valorada especialmente por parte de 
los estudiantes. Aunque el uso de ordenadores portátiles entre los estudiantes de Ingeniería Informática de nuestra universidad ha crecido en los últimos años, sólo los estudiantes de cursos superiores suelen utilizar PDAs. La mayoría de estudiantes que utilizaron PDAs en estos dos casos de estudio no se consideraban a sí mismos usuarios expertos, ni siquiera habituales, de este tipo de dispositivos (esto podría cambiar si se extendiera el uso de sistemas como CoMoLE. Algunos tuvieron dificultades en el uso de estos dispositivos debido a las características de su interfaz de entrada y a la aparición de una pequeña barra de desplazamiento horizontal en el navegador web en determinadas actividades.

El resultado más importante de la evaluación de este trabajo es que los alumnos consideraron los dos entornos desarrollados sobre el sistema CoMoLE. Muy útiles para dar soporte a nuevas maneras de aprendizaje y afrontar el estudio de una materia de una forma distinta. En este sentido, su estudio fue más atractivo, ya que realizaron actividades de distintos tipos desde distintos lugares y diferentes momentos. A la mayoría de los estudiantes, las recomendaciones ofrecidas por el sistema les ayudaron a organizar su tiempo de estudio, especialmente en las situaciones en las que no disponían de mucho tiempo. Su motivación se incrementó, no sólo durante su interacción con el entorno, sino también en discusiones con sus compañeros sobre las explicaciones teóricas, ejemplos y ejercicios que les proponían los entornos. Esto implicó que se les despertara una mayor curiosidad sobre la materia. Los entornos desarrollados para estos casos de estudio tuvieron gran aceptación por parte de los estudiantes. Estos logros demuestran que la recomendación de actividades dependiendo de las características personales de los estudiantes, sus acciones y su contexto tiene sentido en los entornos de aprendizaje móvil adaptativos. Las cifras y datos detallados sobre estos dos casos de estudio se pueden ver publicados en (Martin y Corre, 2009).

\section{EVALUACIÓN DE CURSOS ADAPTATIVOS}

La evaluación de las actividades educativas realizadas a través de entornos virtuales presenta dificultades añadidas sobre la evaluación de la realización de actividades presencialmente. Estas dificultades se ven acrecentadas cuando las actividades son propuestas a través de un sistema adaptativo, donde no todos los estudiantes realizan las mismas actividades ni se les presentan los mismos contenidos. El resultado es que para un profesor es difícil comprobar cuáles son los problemas (desde el punto de vista educativo) que surgen durante la interacción de los estudiantes con el sistema adaptativo o, por ejemplo, si ciertos tipos de estudiantes tuvieron más problemas que otros. 
La evaluación de un curso adaptativo trata de dar respuesta a la pregunta sobre si la adaptación proporcionada tanto a nivel de actividades recomendadas (guía de navegación entre ellas) como a nivel de contenidos presentados fueron los adecuados para todos los perfiles de usuario. Con este objetivo, el método propuesto se basa en detectar situaciones problemáticas que puedan ser consideradas síntomas de problemas, tanto en los contenidos en sí como en los criterios de adaptación. Algunos ejemplos de este tipo de situaciones son: actividades prácticas que un número elevado de estudiantes ha tenido problemas para resolver; un grupo de actividades teóricas que insumen mucho más tiempo del previsto por el profesor; o una actividad concreta que sistemáticamente no puede ser terminada en el tiempo previsto por el sistema.

Así, el método GeSES (Generate-Select-Establish-Select) consiste en analizar los logs generados por sistemas de e-learning (en especial sistemas adaptativos) en busca de estos síntomas de potenciales problemas. Para esto utiliza técnicas de minería de datos, aunque con énfasis en ocultar los detalles de aplicación de dichas técnicas para dar soporte a usuarios con poco o ningún conocimiento técnico que deseen evaluar este tipo de sistemas.

\section{Método GeSES}

La primera propuesta de método para detectar síntomas en cursos adaptativos es Key-node (Bravo et al., 2010). El método consiste en construir árboles de decisión a partir de los logs, donde cada entrada (acción de un usuario con el sistema) se etiqueta como "buena" o "mala" (es decir, situaciones satisfactorias versus situaciones no deseadas, como que el estudiante responda mal a una cuestión práctica). Los árboles construidos no se utilizan para clasificar nuevos casos, sino que se analizan en busca del conocimiento que representan. Así, una rama de un árbol de decisión que lleva a una hoja etiquetada como "mala" posee información sobre actividades problemáticas en el curso, y normalmente también contiene datos sobre los perfiles de los usuarios asociados con ese problema. De esta forma se pueden detectar situaciones donde, por ejemplo, sólo el 30\% de los estudiantes realizan de forma no satisfactoria una determinada actividad, pero ese porcentaje correspondía a la totalidad de estudiantes con un perfil de conocimiento básico.

Pero el método Key-node sufre de problemas de escalabilidad. Así, al crecer los árboles de decisión se torna cada vez más difícil extraer la información de forma que pueda ser entendida y utilizada por un profesor para mejorar el curso. Por este motivo, se desarrolló el método GeSES, que se sirve de una técnica que mejora 
los resultados de un árbol de decisión, las reglas de decisión, y en particular las del algoritmo C4.5 (Quinlan, 1993). Después de las sucesivas mejoras de la propuesta inicial de este método se llegó a la propuesta final, que se puede resumir en las siguientes etapas:

- Fase1: Fase de limpieza. Eliminar los datos de interacción que no se consideren relevantes y establecer la variable de selección. Esta variable se utiliza para etiquetar cada entrada ("buena" o "mala"). Por ejemplo, sería posible evaluar las puntuaciones obtenidas por los estudiantes al realizar las actividades, el tiempo empleado en realizarlas, o la frecuencia de consulta a la tabla de contenidos. Una limitación del algoritmo de aprendizaje utilizado es que la variable que etiqueta cada caso debe ser discreta. Así, si se quieren considerar como característica de evaluación las puntuaciones obtenidas en una determinada actividad (notas numéricas), se debe generar una variable sintética nominal que refleje esta información (por ejemplo, una variable "éxito", cuyos valores posibles serían "sí" y "no", dependiendo de si la puntuación alcanza cierto nivel mínimo). El resultado de esta fase es una tabla con los perfiles de los estudiantes, sus datos de interacción y la variable de evaluación. La información contenida en esta tabla constituye los datos de análisis.

- Fase 2: Generación de las reglas de decisión. Consiste en la aplicación del algoritmo $\mathrm{C} 4.5$ a los datos de análisis para generar las reglas de decisión. Los parámetros para este algoritmo son:

o Atributos: características del perfil del estudiante; nombre, propiedades y relaciones de la actividad (si es una actividad compuesta o no, cuál es la superactividad de la que ésta forma parte - si fuera el caso- conceptos relacionados con la actividad, etc.).

o Variable de clasificación o clase: variable de evaluación.

El resultado de esta fase es un conjunto de reglas de decisión, cada una con su correspondiente precisión, y un ranking de reglas con los siguientes parámetros asociados: size, error, used y class. Estos parámetros describen la calidad de las reglas; el primero responde al tamaño de la regla, es decir, el número de condiciones que hay en la parte izquierda de ésta. El segundo hace referencia a la tasa de error de la regla (porcentajes de casos mal clasificados respecto de los casos bien clasificados). El siguiente indica la cobertura de la regla (es decir, el número total de casos a los que se aplica). El último parámetro se refiere a la clase a la que hace relación la regla, es decir, la parte derecha de la regla. 
- Fase 3: Seleccionar las reglas relevantes para la característica de evaluación. Seleccionar, del conjunto de reglas de decisión, aquellas en las que la variable de evaluación cumpla la característica que se quiere evaluar. En el ejemplo de las puntuaciones, se seleccionarían las reglas de la clase "no", ya que éstas indican que no se resolvió el ejercicio correctamente.

- Fase 4: Establecer la frontera de selección. Un problema asociado con los algoritmos de generación de reglas de clasificación es que, en la mayoría de las situaciones, se genera un número de reglas muy elevado. La mayor parte de las veces las reglas no son relevantes para el profesor, porque, por ejemplo, afectan a una porción muy pequeña de los estudiantes. Sin embargo, la gran cantidad de reglas puede dificultar, e incluso impedir, que el profesor pueda identificar las reglas significativas. Por este motivo, el método presentado incluye criterios para ordenar las reglas por importancia y luego presentar al profesor sólo las que cumplan determinados criterios de significancia. Por ello, es necesario establecer un punto a partir del cual se considera que una regla es relevante, y reducir de esta forma la información mostrada al profesor. Este punto es la frontera de selección, que corresponde al tercer cuartil, de forma que:

o Frontera: tercer cuartil, Q3, respecto de los conceptos: used, size y error. Se seleccionaron estos tres conceptos, ya que éstos contienen la información necesaria para decidir si una regla es relevante o no.

- Fase 5: Seleccionar las mejores reglas. Esta fase está dividida en tres etapas: generación de la precisión, asignación de pesos a las reglas y selección de las reglas.

o Generación de la precisión. Se genera el parámetro precision a partir del parámetro error, pues precisión $=100$ - error, ya que el error está expresado en porcentajes.

o Asignación de pesos a las reglas. En primer lugar, se utiliza una modificación de la función sigmoidal para la asignación de pesos para cada uno de los conceptos used, size y precisión:

$$
W_{c}(x)=\frac{1}{1+\frac{1}{4} * e^{-\frac{x-Q_{c, e}}{\frac{Q c, 3-Q c, 1}{2}}}}
$$


La ecuación contiene los siguientes elementos: wc hace referencia al peso que obtiene el concepto c para la regla, $\mathrm{Q}_{\mathrm{c}, 3} \mathrm{y}_{\mathrm{c}, 1}$ son el tercer y primer cuartil de la tabla de ranking respecto al concepto c, y x es la observación. Una observación es el valor del concepto relacionado con la regla; por ejemplo si la regla tuviera una cobertura de 1400 casos, la observación sería 1400 . El peso total de cada regla se obtiene sumando los pesos de los conceptos, ponderados de acuerdo a la importancia de cada uno de los parámetros, siendo esta ponderación un número entre o y 1 . Hay que indicar que el profesor tiene la libertad de fijar la importancia de estos conceptos, según lo que considere adecuado.

$$
\mathrm{Wr}=\mathrm{w}_{\text {used }} * \mathrm{~s}_{\text {used }} *+\mathrm{w}_{\text {size }} *+\mathrm{w}_{\text {precisión }} * \mathrm{~s}_{\text {precisión }}
$$

Donde, $\mathrm{W}_{\mathrm{r}}$ es el peso total de la regla $\mathrm{r}, \mathrm{W}_{\mathrm{c}}$ es el peso respecto al concepto $\mathrm{c}$ $(c \in\{$ used, size, precisión $\}), \mathrm{y}_{\mathrm{c}}$ es la importancia que tiene $c\left(s_{\mathrm{r}} \in[0,1]\right.$.

o Selección de las reglas. Se ordenan las reglas respecto al peso total $\left(\mathrm{W}_{\mathrm{r}}\right)$ y se seleccionan aquellas que mayores pesos han obtenido.

\section{APLICACIÓN DE GESES PARA EVALUAR UN CURSO EN COMOLE}

Con el objetivo tanto de probar el método GeSES con datos de usuarios reales, como de evaluar uno de los entornos de enseñanza accesibles a través de CoMoLE, se efectuó un análisis de los logs correspondientes a los entornos de enseñanza puestos a disposición de los estudiantes. Esta sección describe la estructura de los datos analizados, los pasos seguidos y los resultados obtenidos.

\section{Descripción de los logs}

Los datos fueron recogidos durante el desarrollo de dos asignaturas de los estudios de Ingeniería Informática impartidos en la Escuela Politécnica Superior de la Universidad Autónoma de Madrid durante el segundo cuatrimestre del curso 2007/o8. Corresponden a las interacciones generadas por los estudiantes de la asignatura de "Sistemas Operativos I" (SO1), mientras realizaban actividades ofrecidas por el sistema CoMoLE para reforzar sus conocimientos sobre esta materia. El sistema realizó recomendaciones de acuerdo a: los estilos de aprendizaje de los estudiantes, las actividades que fueron realizadas por ellos, los resultados obtenidos en las actividades, el contexto en que se encontraban en cada momento (dispositivo utilizado, tiempo disponible, localización física) y los tiempos recomendados para la realización de algunas actividades. El número de estudiantes que realizó las actividades fue 131, generando un total de 3610 interacciones. 
CoMoLE. genera dos ficheros XML para cada estudiante: uno para almacenar su perfil y otro para guardar sus interacciones. Con el objetivo de generar un gran fichero de logs con toda la información sobre todos los estudiantes fue necesario procesar cada uno de los ficheros de cada estudiante, seleccionando las partes útiles para este experimento. Así, el gran fichero generado contiene información relativa a los perfiles de los estudiantes, sus contextos, las actividades realizadas en cada contexto y los resultados obtenidos. Cada uno de los registros (entradas) del fichero de logs contiene las siguientes variables:

- Variables que describen el perfil: se almacenan las variables relativas al identificador del estudiante y las componentes del estilo de aprendizaje modelo de Felder-Silverman (Felder y Silverman, 1988).

IdUser: identificador del estudiante en el sistema.

o Visual: dimensión visual/verbal del modelo de estilos de aprendizaje de Felder-Silverman. Esta dimensión está relacionada con la forma en la que los contenidos son mostrados. Puede tener dos valores: " $y$ " (indica mayor dimensión visual que verbal) y "n" (indica mayor dimensión verbal que visual).

o Sequential: dimensión sequential/global del modelo de Felder-Silverman. Esta dimensión se refiere a la manera en la que los estudiantes entienden la información. Dos valores son posibles: "y" (indica mayor dimensión sequential que global) y "n” (indica mayor dimensión global que sequential).

o Active: dimensión active/reflexive del modelo anterior. Esta dimensión indica la manera preferida de los estudiantes para procesar la información. Dos valores están disponibles: "y" (más active que reflexive) y "n" (más reflexive que active).

o Sensitive: dimensión sensitive/intuitive del modelo de Felder-Silverman. Esta dimensión indica la forma en la que los estudiantes perciben la información. Puede tener dos valores: "y" (más sensitive que intuitive) y "n" (más intuitive que sensitive). 
- Variables respecto al contexto:

o Device: tipo de dispositivo en el que las actividades son presentadas a los estudiantes. En los experimentos realizados se contemplaron dos tipos de dispositivo posibles: PC y PDA.

o Location: lugar en el que se encuentra el estudiante al realizar las actividades. En este caso, existen cuatro posibles lugares: home, laboratory, class y others.

o TimeUser: tiempo del que dispone el estudiante para realizar actividades en una sesión. El estudiante indica este tiempo al principio de la sesión. Valores posibles para esta variable son los siguientes: 10 minutos, 20 minutos, 30 minutos, 1 hora, entre 1 y 2 horas, y más de 2 horas.

- Variables que describen la actividad:

o NameAct: identificador de la actividad de aprendizaje.

o Type: tipo de actividad. Hay cinco tipos de actividades disponibles en este curso: teóricas, ejemplos, test de auto-evaluación, ejercicios de respuesta corta con texto libre y actividades colaborativas.

o Finalization: indica si la actividad se terminó o no. Dos valores son posibles: "F" (actividad finalizada) y "N" (actividad no finalizada).

o Score: almacena el resultado obtenido en la actividad cuando ésta ha sido finalizada. Es una variable continua que toma valores entre o (peor puntuación que se puede obtener) y 10 (mejor puntuación).

o Grade: variable generada de forma sintética (no por CoMoLE) a partir de la variable score. Puede recibir dos valores: LOW (si $0<=$ score $<$ ) y HIGH (si $5<=$ score $<10$ ).

\section{Análisis con el método GeSES}

Como se explicó anteriormente, el método GeSES consta de cinco fases. A continuación se describe cómo se aplicó el método al entorno de enseñanza indicado: 
- Fase 1: Fase de limpieza. Se seleccionaron las interacciones correspondientes al curso de 2007/o8 en la asignatura SO1, y se diseñó la tabla de logs que contiene 3610 filas (logs) y 13 columnas (variables). Esta tabla constituye los datos de análisis, y la variable de evaluación asignada fue la variable grade. La tabla 4.1 muestra un análisis estadístico de la distribución de los datos, mostrando cada variable y su frecuencia absoluta. Así, se muestra que la variable visual tiene dos valores posibles: "y" y "n", siendo la frecuencia del primero de 3140, frente a 470. Dicho de otro modo, hay una mayor proporción de estudiantes visuales que verbales. La misma interpretación puede darse de las otras tres dimensiones del estilo de aprendizaje. Existe mayor número de estudiantes secuenciales que globales, mayor proporción de reflexivos que de activos y mayor proporción de sensitivos que intuitivos. Se observa que el dispositivo más utilizado fue el "PC", que hubo tres tipos de localizaciones, aunque la más frecuente fue realizar las actividades en "casa". Respecto a los tipos de actividades disponibles en este entorno existen tres tipos: test, short_answer y collaborative, correspondientes a tests de autoevaluación, preguntas de respuesta corta, y actividades colaborativas. Fundamentalmente, los estudiantes realizaron actividades de los dos primeros tipos. Para la variable grade, se observa que hubo mayor proporción de valores LOW que de HIGH, indicando que los resultados obtenidos en la realización de las actividades fueron más bien bajos, lo cual puede ser signo de situaciones problemáticas. El número de actividades disponibles fue 46. Sus nombres aparecen en la última fila de la tabla 1. Se sigue la siguiente notación para indicar los nombres de las actividades:

\{nombre-actividad\}(\{número-actividad-inicial\}-\{número-actividad-final\}) o \{nombre-actividad\}\{número-actividad\}.

Por ejemplo, el primer grupo de actividades es PagSimpleTest(1-3), este nombre indica que las actividades disponibles son PagSimpleTest1, PagSimpleTest2 y PagSimpleTest3.

\begin{tabular}{|l|c|c|}
\hline Variable & Valores & Instancias \\
\hline \multirow{2}{*}{ Visual } & $\mathrm{Y}$ & 3140 \\
& $\mathrm{~N}$ & 470 \\
\hline \multirow{2}{*}{ Sequential } & $\mathrm{Y}$ & 2670 \\
& $\mathrm{~N}$ & 940 \\
\hline \multirow{2}{*}{ Active } & $\mathrm{Y}$ & 1364 \\
& $\mathrm{~N}$ & 2246 \\
\hline
\end{tabular}




\begin{tabular}{|l|c|c|}
\hline \multirow{2}{*}{ Sensitive } & $\mathrm{Y}$ & 3181 \\
& $\mathrm{~N}$ & 429 \\
\hline \multirow{2}{*}{ Device } & PC & 3496 \\
& PDA & 114 \\
\hline \multirow{3}{*}{ Location } & Home & 3042 \\
& Lab & 68 \\
\hline \multirow{3}{*}{ Type } & Others & 500 \\
\hline \multirow{2}{*}{ Grade } & Test & 2306 \\
& short_answer & 1235 \\
& collaborative & 69 \\
\hline \multirow{3}{*}{ nameAct } & LOW & 2799 \\
& HIGH & 811 \\
\hline & PagSimpleTest(1-3), PagSimpleRptaLibre(1-2), SegSimpleT- \\
& est(1-2), Mem_Test(1-5), PSRptaLibre(1-2), MV_Test(1-19), \\
& MultiRptaLibre(1-2), PagMV-Ejer(1-7), SegMVEjer, ColSO1, \\
\cline { 2 - 3 } & TablasRptaLibre1, TablasTest2 \\
\hline
\end{tabular}

Tabla 4.1. Distribución de los datos de análisis de los logs de CoMoLE

- Fase 2: Generación de las reglas de decisión. Se aplica el algoritmo C4.5 a los datos de análisis con los siguientes parámetros:

o Atributos: visual, sequential, active, sensitive, device, location, type, nameAct.

o Variable de clasificación: grade.

Después de generar las reglas de decisión con este algoritmo se obtuvieron 43 reglas (13 para la clase LOW y 30 para la clase HIGH). Las reglas se seleccionan a partir de este ranking en las siguientes fases.

- Fase 3: Seleccionar las reglas más relevantes para la característica de evaluación. La variable de evaluación es grade. Como el objetivo del método es detectar signos de baja eficacia y el valor LOW en la variable grade indica que la actividad no se superó adecuadamente, se seleccionaron las reglas de decisión de la clase LOW (13 reglas). La tabla 4.2 muestra las reglas seleccionadas en esta fase. Se observa que la regla que más cobertura presenta es DR104, que es además la que menor error presenta, mientras que la regla DR45 es la de mayor tamaño. 


\begin{tabular}{|c|c|c|c|}
\hline Regla & Size & Error & Used \\
\hline DR104 & 1 & $8.1 \%$ & 1225 \\
\hline DR19 & 1 & $8.5 \%$ & 99 \\
\hline DR30 & 1 & $8.5 \%$ & 99 \\
\hline DR26 & 1 & $9.0 \%$ & 93 \\
\hline DR15 & 2 & $11.8 \%$ & 11 \\
\hline DR57 & 1 & $15.8 \%$ & 81 \\
\hline DR62 & 1 & $17.1 \%$ & 81 \\
\hline DR1 & 2 & $17.2 \%$ & 74 \\
\hline DR54 & 2 & $17.8 \%$ & 418 \\
\hline DR37 & 2 & $18.4 \%$ & 56 \\
\hline DR7 & 1 & $19.7 \%$ & 95 \\
\hline DR20 & 2 & $20.4 \%$ & 54 \\
\hline DR45 & 3 & $22.3 \%$ & 27 \\
\hline
\end{tabular}

Tabla 4.2. Reglas seleccionadas en la fase 3

- Fase 4: Establecer la frontera de selección. Para establecer la frontera se seleccionan las columnas (conceptos) size, error y used de la tabla 2. El resto de las columnas son redundantes, o no contienen información relevante para la fase siguiente. Para los conceptos seleccionados se calculó la frontera de selección: F (tercer cuartil).

\begin{tabular}{|c|c|c|c|}
\hline Estadísticos & Size & Error & Used \\
\hline Suma & 20 & 194.6 & 2413 \\
\hline F & 2 & 9 & 99 \\
\hline
\end{tabular}

Tabla 4.3. Fronteras de selección para los conceptos

Se observa en la tabla 3 que el tercer cuartil para la cobertura es 99. Este es el punto a partir del cual se valorará más a una regla que a otra.

- Fase 5: Seleccionar las mejores reglas. Esta fase consiste en asignar pesos a cada regla, y seleccionar las que mayor peso total presenten. Es importante destacar que el peso otorgado por cada frontera puede recibir un valor entre o y el peso máximo. En este caso, se ha fijado el peso máximo en el valor 1, y se ha 
considerado que cada uno de los conceptos tenga la misma importancia, i.e., $\mathrm{s}_{\text {used }}$ $=\mathrm{S}_{\text {size }}=\mathrm{S}_{\text {error }}=1$. La tabla 4 muestra los pesos otorgados por la función sigmoidal modificada.

\begin{tabular}{|c|c|c|c|c|}
\hline Regla & $\mathbf{W}_{\text {used }}$ & $\mathbf{W}_{\text {size }}$ & $\mathbf{W}_{\text {precision }}$ & $\mathbf{W}_{\mathbf{r}}$ \\
\hline DR104 & 1 & 0.35 & 0.83 & $\mathbf{2 . 1 8}$ \\
\hline DR54 & 1 & 0.8 & 0.38 & $\mathbf{2 . 1 8}$ \\
\hline DR19 & 0.8 & 0.35 & 0.82 & 1.97 \\
\hline DR30 & 0.8 & 0.35 & 0.82 & 1.97 \\
\hline DR7 & 0.77 & 0.35 & 0.29 & 1.41 \\
\hline DR26 & 0.75 & 0.35 & 0.8 & 1.9 \\
\hline DR57 & 0.63 & 0.35 & 0.48 & 1.47 \\
\hline DR62 & 0.63 & 0.35 & 0.42 & 1.4 \\
\hline DR1 & 0.56 & 0.8 & 0.41 & 1.77 \\
\hline DR37 & 0.35 & 0.8 & 0.35 & 1.5 \\
\hline DR20 & 0.33 & 0.8 & 0.26 & 1.39 \\
\hline DR45 & 0.12 & 0.97 & 0.19 & 1.28 \\
\hline DR15 & 0.06 & 0.8 & 0.69 & 1.55 \\
\hline
\end{tabular}

Tabla 4.4. Pesos otorgados por la función sigmoidal

En la tabla 4 se puede observar que las reglas de decisión con mayor peso son DR104 y DR54, seguidas de las reglas DR30 y DR19. Hay que tener en cuenta que el sistema de pesos puntúa sobre 3, esto quiere decir que el mayor peso total posible es 3. En concreto, el mayor peso total se registra en las reglas DR104 y DR54 con una puntuación de 2,18. En este caso se observa que también cobra especial relevancia el grado de importancia de los conceptos (sc). Es importante recordar que al principio se decidió que todos los conceptos tuvieran el mismo grado de importancia, pero si, por ejemplo, la importancia del concepto tamaño fuera mayor que la del concepto precisión, la regla $\mathrm{DR}_{1}$ sería seleccionada en lugar de las reglas DR19 y DR30.

La tabla 5 muestra las reglas que el método considera más relevantes. La regla DR104 indica que la mayoría de los estudiantes que realizaron actividades de tipo short_answer obtuvieron baja puntuación en estos ejercicios. Y la segunda, DR54, indica que la mayoría de estudiantes activos que realizaron sus actividades en casa, presentaron dificultades en la mayoría de actividades. Estas dos reglas contienen información interesante desde el punto de vista de los profesores, ya que les alerta 
de que es posible que exista un problema con las actividades de tipo short_answer. Por tanto, podría ser que estas actividades no fueran adecuadas para los estudiantes activos que trabajaron en casa. Las dos siguientes reglas indican que pueden existir problemas en actividades concretas. En el caso de la regla DR19, se indica que la actividad Mem_Test2 presentó problemas para la mayoría de estudiantes, pues no existe información sobre el perfil de éstos. Las conclusiones son las mismas para la última regla, pero referidas a la actividad MV_Test1.

\begin{tabular}{|c|l|}
\hline DR104: & type $=$ short_answer \\
\cline { 2 - 2 } & $\rightarrow$ class LOW $[91.9 \%]$ \\
\hline DR54: & $\begin{array}{l}\text { active }=y \\
\text { location }=\text { home }\end{array}$ \\
\hline & $\rightarrow$ class LOW $[82.2 \%]$ \\
\hline DR19: & nameAct $=$ Mem_Test2 \\
\hline & $\rightarrow$ class LOW $[91.5 \%]$ \\
\hline DR30: & nameAct $=$ MV_Test1 \\
\hline & $\rightarrow$ class LOW $[91.5 \%]$ \\
\hline
\end{tabular}

Tabla 4.5. Reglas seleccionadas por el método GeSES como las más relevantes

La tabla 4.6 (tabla de signos) presenta un resumen de los signos detectados con el método GeSES. Esta información sería la que se mostraría al profesor, de manera que éste pueda entender qué problemas han sido detectados sin tener grandes conocimientos de minería de datos. El profesor, consultando esta tabla, puede conocer fácilmente las situaciones problemáticas que han tenido lugar, así como el tipo de estudiantes que las han protagonizado y el contexto en el que se encontraban. Hay que señalar que el término "cualquier" se utiliza para indicar que no existe información relativa a los perfiles en la regla de decisión asociada a la fila de la tabla. Este tipo de situaciones se producen más frecuentemente en las reglas de menor tamaño. 


\begin{tabular}{|l|l|}
\hline \multicolumn{1}{|c|}{ Perfil } & \multicolumn{1}{c|}{ Actividad } \\
\hline cualquier & type $=$ short_answer \\
\hline $\begin{array}{l}\text { active }=y \\
\text { location }=\text { home }\end{array}$ & cualquier \\
\hline cualquier & nameAct $=$ Mem_Test2 \\
\hline cualquier & nameAct $=$ MV_Test1 \\
\hline
\end{tabular}

Tabla 4.6. Tabla de signos

Este ejemplo muestra cómo es posible para un profesor sin conocimientos técnicos específicos, y en particular sin formación en técnicas de minería de datos, extraer de los logs información orientada a mejorar el diseño de su curso adaptativo.

\section{ESTADO DEL ARTE}

\section{Sistemas adaptativos y de recomendación}

Los dispositivos y las tecnologías móviles están cambiando las vías a través de las que se puede difundir el conocimiento (Traxler, 2007). En los últimos años, el aprendizaje con dispositivos móviles ha crecido en visibilidad dentro de contextos educativos, incrementándose el número de conferencias, seminarios y talleres de trabajo tanto nacionales como internacionales relacionados con esta área, como (World, 2010), (International, 2010), etc. El uso de dispositivos móviles se puede combinar con el aprendizaje tradicional con el objetivo de incrementar la motivación de los estudiantes, promover la interacción entre los miembros de los grupos de trabajo, facilitar el desarrollo de ciertas habilidades y dar soporte al aprendizaje constructivista (Zurita et al., 2004). Por tanto, es conveniente desarrollar aplicaciones que utilicen estas nuevas tecnologías combinándolas con entornos tradicionales (Gogh et al., 2006; Chen et al., 2007).

Por ejemplo, en (Verdejo et al., 2006) se presenta una propuesta para dar soporte a actividades de aprendizaje tanto dentro como fuera de la escuela. Los estudiantes utilizan una PDA con conexión GPS para grabar observaciones sobre pájaros en un entorno natural. Cuando los alumnos regresan a clase, debaten, analizan y procesan los datos empíricos recogidos.

En la literatura existen algunos sistemas que adaptan las actividades presentadas a los usuarios y/o a su interacción, considerando algunos rasgos personales, acciones o características del contexto de los usuarios. Sin embargo, es difícil que 
permitan la definición y adaptación de cualquier tipo de rasgo que se desee tener en cuenta. Además, algunos de estos sistemas son específicos para la realización de un determinado tipo de actividad. A continuación se describen algunos de ellos.

En (Bull et al., 2003) se presentan las características de dos sistemas adaptativos orientados al aprendizaje móvil llamados MoreMaths y C-POLMILE. MoreMaths es un entorno de aprendizaje orientado exclusivamente al aprendizaje de las matemáticas, que puede ser utilizado desde ordenadores personales o PDAs. La interacción principal se produce cuando los estudiantes utilizan sus ordenadores personales para estudiar distintos conceptos y realizar ejercicios. Desde las PDAs los estudiantes pueden revisar materiales previos. C-POLMILE es un sistema educativo para el aprendizaje del lenguaje de programación C. Existen dos versiones de este sistema, una para ordenadores personales y otra para PDAs. Ambas se adaptan al contexto del usuario incluyendo factores como su localización física, el dispositivo que está utilizando, si existen distracciones en el entorno, etc. De esta manera, el estudiante puede utilizar la más conveniente dependiendo de su situación. Sin embargo, ambas aplicaciones no permiten el desarrollo de actividades colaborativas y sólo tienen en cuenta dentro de las características personales de los estudiantes su estado actual de conocimientos para realizar la recomendación de las distintas actividades.

JAPELAS (Ogata y Yano, 2004) es un entorno específico para aprender expresiones corteses en japonés, no siendo posible la realización de otro tipo de actividades. Este sistema ofrece a los estudiantes las expresiones más adecuadas dependiendo de su situación (despacho, lugar de encuentro, etc.) y de información personal (género, trabajo, edad, etc).

Sc@ut (Gea et al., 2004) es una plataforma para la comunicación en entornos adaptativos ubicuos orientada a niños con autismo. Esta plataforma permite a los niños autistas comunicarse a través de una PDA con conexión a la red inalámbrica. La plataforma presenta a cada niño una serie de plantillas formadas por dibujos o fotografías relacionadas con posibles acciones (lavarse las manos, comer, etc) o sentimientos (estar feliz, enfadarse, etc). Las imágenes presentadas en cada una de las plantillas que se muestran a cada niño están adaptadas a sus características personales teniendo en cuenta el grado de familiaridad de las imágenes y cuáles les pueden motivar más. Durante el proceso de adaptación, la plataforma tiene en cuenta las capacidades y habilidades de los niños, sus acciones y la localización física donde se encuentran. 
TenseITS (Cui y Bull, 2005) es un entorno orientado exclusivamente al aprendizaje de idiomas utilizando PDAs. Este entorno adapta la interacción al conocimiento individual del estudiante. También adapta aspectos contextuales como; la localización del estudiante, teniendo en cuenta la probabilidad de interrupción; el posible nivel de concentración en ese entorno y el tiempo disponible para el estudio.

En (Yau y Joy, 2007) se presenta una aplicación de enseñanza para ser utilizada en PDA. Esta aplicación adapta las actividades de aprendizaje a cada estudiante en función de su estilo de aprendizaje y del contexto en el que se encuentre (tiempo disponible, localización y nivel de ruido). En función de estos dos criterios recomienda las actividades más adecuadas para un determinado usuario. La aplicación consta de dos módulos de adaptación: uno para procesar los estilos de aprendizaje de los alumnos; y, otro para adaptar las actividades al contexto del usuario utilizando para ello reglas de adaptación. La adaptación de estos dos módulos se realiza únicamente teniendo en cuenta los estilos de aprendizaje de los alumnos, y el contexto en el que se encuentran, no siendo posible la introducción de nuevos rasgos de adaptación.

Por otra parte, los sistemas que trabajan con dispositivos móviles no pueden suministrar grandes tablas de datos o dibujos. Algunos sistemas adaptativos adaptan los contenidos al dispositivo utilizado por los usuarios, como los dos que se describen a continuación. APeLS (Brady et al., 2004) es un sistema adaptativo que personaliza un curso de lenguaje SQL para PDAs. Los contenidos del curso son adaptados en función del dispositivo utilizado. Las versiones de contenidos utilizadas para PDAs modifican el tamaño de las tablas HTML, cambian las imágenes por versiones más reducidas o por texto y quitan u ocultan la información redundante o irrelevante para facilitar la visualización de contenidos. En (Kinshuk, 2004) se presenta cómo mejorar el proceso de aprendizaje adaptando la presentación de contenidos a los estilos de aprendizaje de los alumnos en entornos multiplataforma que soporten tanto la conexión de PDAs como de ordenadores personales. Para realizar la adaptación de contenidos a cada estudiante se tienen en cuenta sus estilos de aprendizaje según el modelo de Felder-Sylverman (Felder y Silverman, 1988). En la presente propuesta, se incluye la posibilidad de tener en cuenta no sólo el dispositivo que los usuarios se encuentran utilizando sino también cualquier característica que se haya tenido en consideración en el modelo de usuario, como por ejemplo la dimensión visualverbal de los estilos de aprendizaje de los usuarios.

Por último, algunos sistemas de recomendación con dispositivos móviles orientados a la enseñanza son WANTIT (Graham et al., 2004) e InLinx (Bighini et al., 2003). WANTIT es un portal adaptativo orientado a dispositivos móviles que 
utiliza técnicas de adaptación de la navegación (guía directa, ocultación de enlaces irrelevantes y ordenación de los enlaces) para personalizarla en función de las características del usuario. Este sistema presenta a los usuarios una lista con las sugerencias más relevantes. La lista se encuentra dividida en tres áreas: enlaces más populares, enlaces más probables y enlaces a páginas previas. Para predecir los enlaces más relevantes, que serán presentados en estas tres áreas de una forma ordenada, se utiliza la información almacenada en el modelo de usuario y en un modelo de Markov. InLinx es un sistema híbrido de recomendación que da soporte a la anotación de libros dentro de una plataforma para el aprendizaje móvil (Bighini et al., 2003). InLinx combina la técnica de recomendación basada en contenidos y el filtrado colaborativo para ayudar a los estudiantes a clasificar la información y guardarla en marcadores. Además recomienda estos documentos a otros estudiantes con intereses similares, de entre un gran volumen de documentos, y se encarga de notificar a los estudiantes nuevos documentos que pueden ser interesantes para ellos.

\section{Análisis de datos en sistemas educativos}

En lo que respecta al análisis de datos en el ámbito de los sistemas e-learning, según el estudio de Romero et al. (Romero, 2007) las técnicas más utilizadas son los algoritmos de clasificación (Arabie et al., 1996) y las reglas de asociación (Agrawal et al., 1993). Además, esta tendencia se sigue manteniendo según el reciente estudio realizado por Bakerr y Yacef (Bakerr y Yacef, 2009) en el que se comparan el número de artículos de cada categoría publicados en las conferencias Educational Data Mining de 2008 y 2009; sin embargo, las técnicas de predicción, donde estos autores encuadran a las técnicas de clasificación y de regresión, están siendo más aplicadas en la actualidad que las técnicas de asociación y clustering. La Minería Web (Web Mining) es el campo utilizado para la evaluación de los sistemas Web. En concreto, la minería de uso de Web (Web Usage Mining) es el campo de la Minería Web encargado del análisis de los datos de interacción. En (Srivastava et al., 2000) definen a la minería de uso de Web como el proceso de aplicar técnicas de la minería de datos al descubrimiento de patrones de uso en los datos Web. Este proceso se divide en tres etapas principales: preprocesamiento, descubrimiento de patrones y análisis de patrones.

Hay que señalar que los trabajos existentes en la literatura realizan en menor o mayor medida cada una de estas etapas en el análisis de logs. Las aplicaciones de la minería de Uso de Web son muy diversas en los sistemas adaptativos orientados a la enseñanza. Según apuntan Romero et al. las principales aplicaciones de esta 
disciplina residen en: creación de sistemas de personalización (Mobasher et al., 2000), sistemas de recomendación (Zaïane, 2006; Talavera y Gaudioso, 2004; Ueno, 2006; Romero, 2006; Khribi et al., 2008), sistemas de detección de outliers y de situaciones problemáticas (Baker et al., 2004; Romero et al., 2004), y sistemas de modificación (Su et al., 2006). La tabla 7 muestra los principales trabajos realizados en la aplicación de la minería de uso de Web y las técnicas utilizadas. Así, por ejemplo (Merceron y Yacef, 2003) se sirve de análisis estadísticos y reglas de asociación para construir un sistema de detección.

\begin{tabular}{|l|l|l|l|}
\hline \multirow{2}{*}{\multicolumn{1}{|c|}{ Técnicas }} & \multicolumn{3}{c|}{ Sistemas } \\
\cline { 2 - 4 } & Recomendación & Detección & Modificación \\
\hline Análisis Estadístico & $\begin{array}{l}\text { (Merceron, 2003) } \\
\text { (Ueno, 2006) }\end{array}$ & \\
\hline Reglas de Asociación & $\begin{array}{l}\text { (Talavera, 2004) } \\
\text { (Zä̈ane, 2006) } \\
\text { (Khribi, 2008) }\end{array}$ & $\begin{array}{l}\text { (Merceron, 2003) } \\
\text { (Ben-Naim, 2009) }\end{array}$ & \\
\hline Clustering & (Ueno, 2006) & & $(\mathrm{Su}, 2006)$ \\
\hline Clasificación & (Romero, 2006b) & (Baker, 2004) & $(\mathrm{Su}, 2006)$ \\
\hline Patrones Secuenciales & & & \\
\hline Algoritmos Evolutivos & & (Romero, 2004) & \\
\hline
\end{tabular}

Tabla 5.1. Técnicas utilizadas en las distintas aplicaciones de la minería de datos

\section{CONCLUSIONES}

Las tecnologías de la información han evolucionado y continúan evolucionando enormemente en los últimos años. Entre otras cosas, estos avances han propiciado que el acceso a Internet desde cualquier sitio a través de dispositivos móviles sea una situación muy común y al alcance de cada vez más personas en nuestra sociedad. Paralelamente, es evidente que uno de los bienes que resultan más escasos en esa misma sociedad es el tiempo, situación que obliga a las personas a intentar sacar el máximo provecho de cualquier rato disponible.

Los sistemas adaptativos son una vía muy adecuada para capitalizar las oportunidades que ofrece la tecnología para facilitar el acceso a la información desde cualquier sitio y permitir a los usuarios aprovechar al máximo el tiempo disponible. En este sentido, es sumamente conveniente contar con sistemas que ofrezcan soporte a la recomendación de actividades a realizar en cada momento en pro de una óptima organización del tiempo disponible. 
Este trabajo demuestra que es posible utilizar mecanismos de recomendación basados en el perfil y el contexto de los usuarios en entornos de aprendizaje móviles reales. La retroalimentación y opiniones recibidas sobre el uso de dos entornos concretos (uno de ellos descrito en este artículo) refuerzan la confianza en la utilidad de este tipo de mecanismos, pues han sido valorados positivamente por los usuarios finales.

El sistema CoMoLE. se puede considerar un sistema de recomendación híbrido, pues integra recomendaciones basadas en reglas definidas por expertos con recomendaciones basadas en la información disponible sobre otros usuarios que interactuaron previamente con el entorno. Esto hace posible utilizar uno o ambos tipos de recomendaciones, según se desee. Para facilitar el uso del primer tipo de recomendación, CoMoLE. incorpora algunos filtros de contexto (reglas) genéricos que pueden ser seleccionados por el responsable del entorno sin tener que especificar reglas. Además, gracias al segundo tipo de recomendaciones, no es necesario especificar ni seleccionar ningún criterio de recomendación en forma de reglas.

Sin embargo, para que un sistema de recomendación de este tipo sea realmente efectivo, no basta con poner a disposición de los usuarios los entornos generados por el sistema, sino que es necesario comprobar si la recomendación de actividades y la adaptación de contenidos se está realizando de manera adecuada. En este trabajo se propone el método GeSES para evaluar entornos de aprendizaje adaptativos. El objetivo principal de este método es facilitar dicha evaluación, de forma que los profesores sin un conocimiento técnico específico sean capaces de evaluar la eficacia de sus entornos. No es fácil para un profesor determinar si la recomendación y adaptación funcionan como él espera para cada usuario, ni si éstas son beneficiosas para los estudiantes. Tal como se muestra a través de un ejemplo de aplicación del método, GeSES representa un importante avance para resolver el problema de la evaluación de entornos de enseñanza adaptativos, aunque aún queda trabajo por hacer para resolver el problema completamente. Al mismo tiempo que se mostró la aplicabilidad del método, el análisis de los logs de CoMoLE. permitió detectar algunos problemas en ciertas actividades de un entorno de enseñanza adaptativo generado dinámicamente por CoMoLE. Estos problemas se muestran al profesor, de modo que éste pueda intervenir añadiendo/eliminando/modificando actividades, contenidos o criterios de recomendación o adaptación.

El método de evaluación propuesto se basa fuertemente en reglas de decisión, y éstas dependen en gran medida de la distribución de los datos de análisis. Por tanto, pueden existir situaciones en las que la eficacia mostrada para la detección del método 
en este artículo se vea disminuida. Cabe mencionar que la aplicación del método al conjunto de datos analizado mostró que hubo cierta pérdida de información en la tabla de signos. Esta pérdida se produjo fundamentalmente por un tamaño pequeño de las reglas de decisión seleccionadas; sin embargo, este problema es subsanable asignando mayor importancia al concepto tamaño frente a los conceptos cobertura y precisión. No obstante, la información contenida en una regla con tamaño pequeño puede ser relevante para el profesor, por lo que el profesor debe ser quién fije el grado de importancia de cada concepto.

La generación de nuevos entornos de enseñanza adaptativos basados en CoMoLE. y su evaluación utilizando GeSES pondrán de manifiesto las bondades de ambos sistemas para generar y evaluar, respectivamente, este tipo de entornos. Dichos entornos han mostrado ser muy útiles hoy en día para facilitar el aprendizaje a usuarios con distintas características y necesidades en distintos contextos, facilitando, mediante la recomendación de actividades y la generación dinámica de espacios de trabajo con herramientas y contenidos adaptados, un mejor aprovechamiento del tiempo.

\section{AGRADECIMIENTOS}

Este trabajo ha sido parcialmente financiado por el Ministerio de Ciencia y Tecnología de España (TIN2007-64718) y la Comunidad Autónoma de Madrid (S2009/TIC-1650).

\section{REFERENCIAS BIBLIOGRÁFICAS}

Agrawal, R.; Imielinski, T.; Swami, A. (1993). Mining association rules between sets of items in large databases. En: Buneman, P.; Jajodia, S. (eds.), Proceedings of ACM SIGMOD Conference on Management of Data, Washington, USA. ACM Press, (207-216).

Arabie, P.; Hubert, L. J.; Soete, G. (1996). Clustering and Classification. World Scientific Publishers.

Baker, R.; Corbett, A.T.; Koedinger, K. R. (2004). Detecting Student Misuse of Intelligent Tutoring Systems. En: Lester, C. J.; Vicari, R. M.; Paraquaçu, F. (eds.), Proceedings of the 7 th International
Conference on Intelligent Tutoring Systems, vol. 3220, Brazil: Springer. (531-540).

Baker, R.; Yacef, K. (2009). The State of Educational Data Mining in 2009: A Review and Future Visions. Journal of Educational Data Mining, vol. 1 (1), (115).

Ben-Naim, D.; Bain, M.; Marcus, N. (2009). A User-Driven and Data-Driven Approach for Supporting Teachers in Reflection and Adaptation of Adaptive Tutorials. En Proceedings of the Second International Conference on Educational Data Mining - EDMo9, Córdoba, Spain, Universidad 
de Córdoba, (21-30).

Bighini, C.; Carbonaro, A.; Casadei, G. (2003). InLinx for document classification, sharing and recommendation. Proceedings of the 3rd IEEE International Conference on Advanced Learning Technologies, 91-95.

Brady, A.; Conlan, O.; Wade, V. (2004). Dynamic Composition and Personalization of PDA-based eLearning - Personalized mLearning. Proceedings of World Conference on E-Learning in Corporate, Government, Healthcare, and Higher Education 2004. Chesapeake, VA: AACE, (234-242).

Bravo, J.; Vialardi, C.; Ortigosa, A. (2010). Using decision trees for improving $\mathrm{AEH}$ courses. Handbook of educational data mining. Chapter 26. Taylor and Francis.

Brusilovsky P.; Kobsa A.; Vassileva J. (1998). Adaptive Hypertext and Hypermedia. Kluwer Academic Publishers, 1-43.

Bull, S.; McEvoy, A. T.; Reid, E. (2003). Learner Models to Promote Reflection in Combined Desktop PC / Mobile Intelligent Learning Environments. En: Bull, S.; Brna, P.; Dimitrova, V. (eds.). Proceedings of the International Workshop on Learner Modelling for Reflection at 11th International Conference on Artificial Intelligent in Education, (199-208).

Carro, R. M. (2001). Un mecanismo basado en tareas y reglas para la creación de sistemas hipermedia adaptativos: aplicación a la educación a través de Internet. Tesis doctoral. Departamento de Ingeniería Informática. Universidad Autónoma de Madrid.

Chen, M.; Yen, J. (2007). An evaluation of learners' satisfaction toward mobile learning. Proceedings of the 6th Conference on WSEAS International Conference on Applied Computer Science, vol. 6. World Scientific and Engineering Academy and Society, Stevens Point, Wisconsin, (382-388).
Cui, Y.; Bull, S. (2005). Context and learner modelling for the mobile foreign language learner. System, vol. 33 (2), (353-367).

Felder, R. M.; Silverman, L. K. (1988). Learning Styles and Teaching Styles in College Science Education. J. College Science Teaching, 23(5), (286-290).

Gea-Megías, M.; Medina-Medina, N.; Rodríguez-Almendros, M. L.; RodríguezFórtiz, M. J. (2004). Sc@ut: Platform for Communication in Ubiquitous and Adaptive Environments Applied for Children with Autism. User-Centered Interaction Paradigms for Universal Access in the Information Society, Lecture Notes in Computer Science, vol. 3196, Springer Berlin / Heidelberg. (5067).

Goh, T.; Kinshuk (2006). Getting Ready For Mobile Learning-Adaptation Perspective. Journal of Educational Multimedia and Hypermedia, vol. 15 (2), Chesapeake, VA: AACE. (175-198).

Graham, P.; Bowerman, C.; Bokma, A. (2004). Adaptive navigation for mobile devices. Learning with mobile devices: research and development. London: LSDA, (61-67).

International Conference on Wireless, Mobile and Ubiquitous Technologies in Education. (2010). [en línea] Disponible en: http://wmute2010.cl.ncu.edu.tw/ (consulta 2010, mayo).

Khribi, M. K.; Jemni, M.; Nasraoui, O. (2008). Automatic Recommendations for e-Learning Personalization Based on Web Usage Mining Techniques and Information Retrieval. En: Díaz, P.; Kinshuk, Aedo, I.; Mora, E. (eds.). Proceedings of the 8th IEEE International Conference on Advanced Learning Technologies. Santander, Spain. IEEE Computer Society, (241245).

Kinshuk, Lin T. (2004). Application of learning styles adaptivity in mobile 
learning environments. Third Pan Commonwealth Forum on Open Learning.

Martín, E.; Carro, R. M. (2009). Supporting the Development of Mobile Adaptive Learning Environments: A Case Study. IEEE Transactions on Learning Technologies, vol. 2 (1), (23-36).

Merceron, A.; Yacef, K. (2003). A web-based tutoring tool with mining facilities to improve learning and teaching. En: Hoppe, U.; Verdejo, F.; Kay, J. (eds.). Proceedings of the 11th International Conference on Artificial Intelligence in Education - AIED. Sydney, Australia. IOS Press, (201-208).

Mobasher, B.; Cooley, R.; Srivastava, J. (2000). Automatic personalization based on Web usage mining. Communication of the Association of Computing Machinery (ACM), vol. 43 (8), (142-1519).

Ogata, H.; Yano, Y. (2004). Context-aware support for computer-supported ubiquitous learning. Proceedings of IEEE International Workshop on Wireless and Mobile Technologies in Education, Taiwan. IEEE Computer Society, (2734).

Quinlan, J.R. (1993). C4.5: programs for machine learning. Morgan Kaufmann Publishers, Inc., 17th Edition.

Romero, C.; Porras, A. R.; Ventura, S.; Hervás, C.; Zafra, A. (2006). Using sequential pattern mining for links recommendation in adaptive hypermedia educational systems. En: Méndez-Vilas, A.; Solano, A.; Mesa, J. A.; Mesa, J. (eds.), Proceedings of the 4th International Conference on Multimedia and Information and Communication Technologies in Education - Current Developments in Technology-Assisted Education (Technological Issues), vol. 2, Sevilla, Spain. (1016-1020).

Romero, C.; Ventura, S. (2007). Educational Data Mining: a Survey from 1995 to 2005. Expert Systems with Applications, vol. 33 (1)(135-146).

Romero, C.; Ventura, S. (eds.) (2006). Data Mining in e-Learning. WIT Press, Southampton, UK.

Romero, C.; Ventura, S.; Barnes, T. Desmarais, M. (eds.) (2009). Proceedings of the Second International Conference on Educational Data Mining - EDMo9, Córdoba, Spain: Universidad de Córdoba.

Romero, C.; Ventura, S.; de Bra, P. (2004). Knowledge Discovery with Genetic Programming for Providing Feedback to Courseware Authors. User Modeling and User-Adapted Interaction, vol. 14 (5), (425-464).

Srivastava, J.; Cooley, R.; Deshpande, M.; Tan, P. (2000). Web Usage Mining: Discovery and Applications of Usage Patterns from Web Data. SIGKDD Explorations, vol. 1(2), (12-23).

Su, J. M.; Tseng, S. S.; Wang, W.; Weng, J. F. (2006). Learning Portfolio Analysis and Mining for SCORM Compliant Environment. Educational Technology and Society, vol. 9 (1), (262-275).

Talavera, L.; Gaudioso, E. (2004). Mining Student Data to Characterize Similar Behavior Groups in Unstructured Collaboration Spaces. En Proceedings of the Workshop on Artificial Intelligence Methods in Computer-Supported Collaborative Learning (CSCL) held in conjunction with the 16th European Conference on Artificial Intelligence ECAI, Valencia, Spain. (17-23).

Traxler, J. (2007). Defining, Discussing and Evaluating Mobile Learning: the moving finger writes... The International Review of Research in Open and Distance Learning, vol. 8 (2). [en línea] Disponible en: http://www.irrodl.org/index.php/ irrodl/article/view/346/882 (consulta 2010, Mayo).

Ueno, M. (2006). Online outlier detection of learners' irregular learning processes. En: Romero \& Ventura (eds.), Data 
Mining in e-Learning, (261-277).

Verdejo, M. F.; Celorrio, C.; Lorenzo, E.; Sastre-Toral, T. (2006). An educational networking infrastructure supporting ubiquitous learning for school students. Proceedings of 6th IEEE International Conference on Advanced Learning Technologies, (174-178).

Weber, G.; Specht, M. (1997). User modeling and adaptive navigation support in WWW-based tutoring systems. Proceedings of User Modeling '97, (289300).

Witten, I. H.; Frank, E. (2000). Data Mining: practical machine learning tools and techniques with java implementations. Morgan Kaufmann Publishers Academic Press.

World Conference on Mobile and Contextual Learning (2010). [en línea] Disponible en: $\quad$ http://www.mlearn2010.org/ (consulta 2010, Mayo).

Yau, J.; Joy, M. (2007). A Context-aware and Adaptive Learning Schedule framework for supporting learners' daily routines. Proceedings of the Mobile Communications and Learning Workshop, (31-37).

Zaïane, O. R. (2006). Recommender System for e-Learning: Towards Non-Instructive Web Mining. En: Romero \& Ventura (eds.), Data Mining in e-Learning, (7996).

Zurita, G.; Nussbaum, M. (2004). A Constructivist Mobile Learning Environment Supported by a Wireless Handheld Network. Journal of Computer Assisted Learning, vol. 20, (235-243).

\section{PERFIL ACADÉMICO Y PROFESIONAL DEL AUTORES}

Alvaro Ortigosa es doctor por la Universidad Autónoma de Madrid desde el año 2000, y actualmente es profesor contratado doctor en el Departamento de Ingeniería Informática de dicha universidad. Sus áreas de interés son los sistemas basados en modelos de usuario y el uso de técnicas de inteligencia artificial para dar soporte a sistemas adaptativos y de recomendación. Más información en_www.eps. uam.es/ ortigosa.

E-mail: alvaro.ortigosa@uam.es

Javier Bravo, recientemente ha depositado su tesis doctoral en la Universidad Autónoma de Madrid, cuya defensa está prevista para julio de 2010. Actualmente está trabajando en el proyecto eMadrid como investigador contratado. Sus líneas de investigación abarcan el amplio campo de la evaluación de sistemas e-Learning. Mayor información sobre su trayectoria académica y profesional se puede encontrar en http://www.eps.uam.es/ jbravo.

E-mail: javier.bravo@uam.es

Estefanía Martín obtuvo su grado de doctora en la Universidad Autónoma de Madrid en el año 2008. En la actualidad es profesor contratado doctor en el Departamento de Lenguajes y Sistemas Informáticos I de la Universidad Rey Juan 
Carlos. Sus líneas de investigación son la hipermedia adaptativa, el aprendizaje colaborativo y el aprendizaje móvil. Se puede encontrar más información en http:// www.escet.urjc.es/ emartin

E-mail: estefania.martin@urjc.es

Rosa M. Carro es doctora por la Universidad Autónoma de Madrid desde el año 2001 y profesora titular en el Dpto. de Ingeniería Informática de dicha universidad. Sus líneas de investigación se centran en hipermedia adaptativa, modelado de usuario, sistemas colaborativos y evaluación. Ha organizado varios workshops y forma parte del comité de programa de numerosos congresos internacionales, siendo también revisora para varias revistas de IEEE. Ha publicado más de 80 artículos. Más información en http://www.eps.uam.es/ rcarro

E-mail: rosa.carro@uam.es

DIRECCIÓN DE LOS AUTORES:

\author{
Alvaro Ortigosa Juárez, \\ Departamento de Ingeniería Informática, \\ Universidad Autónoma de Madrid, \\ Campus de Cantoblanco, \\ Francisco Tomás y Valiente 11, office B-313, \\ Madrid 28049, España \\ Javier Bravo Agapito, \\ Departamento de Ingeniería Informática, \\ Universidad Autónoma de Madrid, \\ Campus de Cantoblanco, \\ Francisco Tomás y Valiente 11, \\ office B-207, Madrid 28049, España \\ Estefanía Martín Barroso, \\ Departamento de Lenguajes \\ y Sistemas Informáticos I, \\ Universidad Rey Juan Carlos, \\ Despacho 2027, \\ Ampliación Del Edificio De Rectorado, \\ c/Tulipán s/n, Móstoles 28933, España.
}


Rosa M. Carro Salas, Departamento de Ingeniería Informática, Universidad Autónoma de Madrid, Campus de Cantoblanco, Francisco Tomás y Valiente 11, office B-318, Madrid 28049, España.

Fecha de recepción del artículo: 10/05/10

Fecha de aceptación del artículo: 17/07/10 